\title{
Publisher Correction: Multi-trait analysis of genome-wide association summary statistics using MTAG
}

Patrick Turley, Raymond K. Walters @D, Omeed Maghzian, Aysu Okbay, James J. Lee (D), Mark Alan Fontana, Tuan Anh Nguyen-Viet, Robbee Wedow (D), Meghan Zacher, Nicholas A. Furlotte, 23andMe Research Team, Social Science Genetic Association Consortium, Patrik Magnusson (D), Sven Oskarsson, Magnus Johannesson (D), Peter M. Visscher (1D, David Laibson, David Cesarini, Benjamin M. Neale and Daniel J. Benjamin (D)

Correction to: Nature Genetics https://doi.org/10.1038/s41588-017-0009-4, published online 1 January 2018.

In the HTML version of this article initially published, the author groups '23andMe Research Team' and 'Social Science Genetic Association Consortium' appeared at the end of the author list but should have appeared earlier in the list, after author Nicholas A. Furlotte. The errors have been corrected in the HTML version of the article.

Published online: 30 May 2019

https://doi.org/10.1038/s41588-019-0444-5

\section{Publisher Correction: Molecular and functional variation in iPSC-derived sensory neurons}

Jeremy Schwartzentruber (iD, Stefanie Foskolou, Helena Kilpinen, Julia Rodrigues, Kaur Alasoo (D), Andrew J. Knights, Minal Patel, Angela Goncalves, Rita Ferreira, Caroline Louise Benn, Anna Wilbrey, Magda Bictash, Emma Impey, Lishuang Cao, Sergio Lainez, Alexandre Julien Loucif, Paul John Whiting, HIPSCI Consortium, Alex Gutteridge and Daniel J. Gaffney

Correction to: Nature Genetics https://doi.org/10.1038/s41588-017-0005-8, published online 11 December 2017.

In the version of this article initially published, the author group 'HIPSCI Consortium' appeared at the end of the author list but should have appeared in the third-to-last position. The error has been corrected in the HTML and PDF versions of the article.

\section{Publisher Correction: Genome-wide association meta-analysis of individuals of European ancestry identifies new loci explaining a substantial fraction of hair color variation and heritability}

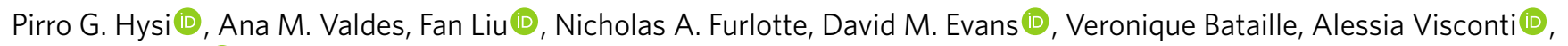
Gibran Hemani (D), George McMahon, Susan M. Ring, George Davey Smith, David L. Duffy, Gu Zhu, Scott D. Gordon, Sarah E. Medland (D), Bochao D. Lin, Gonneke Willemsen, Jouke Jan Hottenga, Dragana Vuckovic, Giorgia Girotto, Ilaria Gandin, Cinzia Sala, Maria Pina Concas, Marco Brumat (1), Paolo Gasparini, Daniela Toniolo, Massimiliano Cocca (D), Antonietta Robino, Seyhan Yazar, Alex W. Hewitt (1), Yan Chen, Changqing Zeng, Andre G. Uitterlinden, M. Arfan Ikram (1), Merel A. Hamer, Cornelia M. van Duijn, Tamar Nijsten, David A. Mackey, Mario Falchi, Dorret I. Boomsma,

Nicholas G. Martin, The International Visible Trait Genetics Consortium, David A. Hinds (1D, Manfred Kayser (D) and Timothy D. Spector

Correction to: Nature Genetics https://doi.org/10.1038/s41588-018-0100-5, published online 16 April 2018.

In the HTML version of this article, the author group 'The International Visible Trait Genetics Consortium' was duplicated and appeared at the end of the author list but should have appeared only once, in the fourth-to-last position. The error has been corrected in the HTML version of the article. 